\title{
Nachträge und Berichtigungen zum Verzeichnis der Coleopteren des Monte Gargano.
}

\author{
Von Karl Holdhaus.
}

Im Jahre 1911 habe ich ein Verzeichnis der Käfer und Landschnecken des Monte Gargano ${ }^{1}$ ) veröffentlicht und hiebei im besonderen die auffallenden Beziehungen der Fauna dieses Gebirgsstockes zu jener der Balkanhalbinsel klarzulegen versucht. Im folgenden seien einige Berichtigungen und Nachträge zu dieser Arbeit gegeben:

\section{Berichtigungen.}

Staphylinus brunnipes F. ist bisher vom Monte Gargano nicht bekannt. Die im Verzeichnis pag. 439 als St. brunnipes angeführte Form gehört nicht zu dieser Art, sondern ist nach dem derzeitigen Stande unserer Kenntnisse als Staphylinus pullus Hochh. (simulator Epp.) zu bestimmen. St. pullus ist eine bisher wenig untersuchte Art, deren Zerlegung in mehrere Spezies sich bei genauerem Studium vielleicht als notwendig erweisen dürfte. Neuraphes planiceps Reitt. kommt am Monte Gargano nicht vor. Die infolge eines bedauerlichen und kaum zu entschuldigenden Gedächtnisfehlers im Verzeichnis p. 440 irrtümlich als $N$. planiceps angeführte Art ist in Wirklichkeit mit $N$. angulatus M. u. K. entweder identisch oder sehr nahe verwandt. Es bedürfte genauerer und zeitraubender Untersuchungen, um hierüber volle Klarheit zu gewinnen.

N a chträge.

Carabus intricatus L. Das am Monte Gargano bei S. Angelo aufgefundene Exemplar des $C$. intricatus gehört zur subsp. Leonii Born, welche mit der calabrischen subsp. silaensis Haury zunächst verwandt ist (det. Born). C. intricatus Leonii ist beschrieben aus der Basilicata.

Phyllobius sinuatus F. Die Determination der fraglichen Exemplare als $P h$. simuatus ist richtig und die Angabe Schilskys (Käf. Eur., Heft 47, 5), daß Ph. Paganettii Schils. am Monte Gargano vorkommt, entspricht nicht den Tatsachen. Herr Kustos Apfelbeck, welcher die von Schilsky als $P h$. Paganettii

$\left.{ }^{1}\right)$ K. Holdhaus, Über die Coleopteren- und Molluskenfauna des Monte Gargano (unter besonderer Berücksichtigung der Adriatisfrage). Denkschriften der mathem.-naturwiss. Klasse der kais. Akademie der Wissenschaften in Wien, LXXXVII. Bd., 1911, pag. 431-465.

Wien. Entom. Zeitung, XXXIV. Jhg., Heft VHI-X, Festschr. f. Reitter (22. Okt. 1915). 
bestimmten Exemplare vom Monte Gargano in der Coll. Leonhard und im Berliner königl. Museum untersuchte, konnte deren zweifellose Identität mit dem echten $P h$. sinuatus feststellen. Ph. Paganettii ist nach den Feststellungen von Kustos A pfelbeck eine gute Art, die aber bisher nur aus dem zentralen Apennin (Castel di Sangro und Camerata nuova) sowie aus den Murgien (S. Basilio) bekannt ist.

Stomodes Amorei Desbr. ist nach den sorgfältigen Untersuchungen von H. Wagner (Wiener Ent. Zeitg. 1912, pag. 269) identisch mit St. tolutarius Boh. Die geographische Verbreitung der Art ist nach Wagner folgende: Italien (Monte Gargano; Cer(hio), Kroatien (Velebit), Dalmatien, Griechenland (Parnaß), Krim, Armenien. Hingegen ist die Provenienzangabe Amur wohl sicher falsch. Der Käfer ist ungeflügelt.

Ich unterließ es absichtlich, in die Arbeit über die Käferfauna des Monte Gargano Neubeschreibungen aufzunehmen, da die Zeitschrift, in der die Arbeit veröffentlicht wurde, vielen Entomologen etwas schwer zugänglich sein dürfte. Von den am Monte Gargano aufgefundenen neuen Arten sind die meisten inzwischen bereits beschrieben worden. Ich gebe im folgenden die Zitate:

Xantholinus (Typhlodes) garganicus Bernh. Münchener Kol. Zeitschr., III (1906-1908), p. 323.

Sipatia Lconhardi Bernh., ibid., pag. 325.

Anthicus Leonhardi Krek. Wiener Ent. Zeitg. 1913, pag. 229.

Otiorrhynchus spalatrensis transadriaticus (K. Dan. in litt.) Reitt.

Wiener Ent. Zeitg. 1913, pag. 40. Die Form wurde sowohl von Dr. K. Daniel als auch von Solari und A pfelbeck auf Grund reicheren Untersuchungsmaterials als Rasse des $O t$. spalatrensis erkannt.

Otiorrhynchus indefinitus (Solari in litt.) Reitt. Wiener Ent. Zeitg. 1912, pag. 126.

Otiorrhynchus Jovis Holdhausi Sol. Wiener Ent. Zeitg. 1913, pag. 121.

Eine neue Scydmaenidenart vom Monte Gargano sei im folgenden beschrieben :

\section{Stenichnus Hilfi nov. spec.}

Mit Stenichnus Helferi Schaum nahe verwandt, von dieser Art durch etwas größere Basalgrübchen des Pronotums, rudimentäre Flügel, etwas breiter ovale, stärker gewölbte Flügeldecken mit viel 
feinerer Punktierung und durch die beim o nach oben ausgesprochener winkelig erweiterten Vorderschenkel verschieden. ${ }^{1}$ )

Schwarz, oft mit einem Stich ins Bräunliche, auf der Oberseite wie bei Stenichnus Helferi mit mäßig langer, gelblicher Behaarung, die Fühler, Taster und Beine gelbbraun. Kopf wie bei Stenichnus Helferi gebildet, an den Fühlern das siebente Glied in seiner größten Breite kaum merklich breiter als lang, das achte Glied deutlich quer, das neunte und zehnte Glied um mehr als ein Drittel breiter als lang. Pronotum ungefähr so breit wie lang, an der Basis mit sechs großen tiefen Grübchen. Flügeldecken oval, ziemlich stark gewölbt, an den Seiten stark und gleichmäßig gerundet, mit deutlicher, bereits unter sehr starker Lupenvergrößerung wahrnehmbarer Chagrinierung und feiner, wenig deutlicher und wenig dichter Punktierung, mit kräftigem Skutellargrübchen, das sich nach rückwärts in eine sehr seichte, das vordere Drittel der Flügeldeckenlänge durchziehende Furche fortsetzt, innerhalb der kräftigen Schulterfalte mit tiefer, länglicher Intrahumeralimpression. Die Behaarung der Flügeldecken von derselben Länge wie bei Stenichnus Helferi, schräg abstehend. Flügel rudimentär. Beim o die Vorderschenkel nach oben etwas stärker er weitert als bei Stenichnus Helferi, kurz vor der Spitze am breitesten, ihr Oberrand daselbst einen verrundeten rechten Winkel bildend. Mittel- und Hinterbeine ohne Sexualauszeichnung. L on g. : $1 \cdot 3-1.4 \mathrm{~mm}$.

Monte Gargano (Apulien), am Abhang der Valle Carbonara unterhalb Monte S. Angelo in Gesellschaft von Stenichnus Helferi in Mehrzahl gesammelt. Ich widme diese Art meinem Reisebegleiter Herrn Moritz Hilf, welcher die Coleopterenfauna des Monte Gargano in erfolgreichster Weise explorierte.

Ich benütze die Gelegenheit, im folgenden noch eine zweite Stenichnus-Art zu beschreiben, welche gleichfalls aus dem südlichen Italien stammt.

\section{Stenichnus egregius nov. spec.}

Vermutlich mit dem mir in natura nicht vorliegenden Stenichnus angustissimus Peyerimhoff (L'Abeille, XXX, 1901, pag. 58) aus den Basses-Alpes zunächst verwandt, von dieser Art durch bedeutendere Körpergröße, rötlichbraune Färbung, das Vorhandensein deutlicher,

1) Die Artgruppe des Stenichnus Helferi ist übrigens noch keineswegs geklärt und es ist zu vermuten, daß st. Helferi selbst eine Mischart ist, deren korrekte Zerlegung wohl nur auf Grund umfangreicher anatomischer Untersuchungen möglich wäre.

Wien. Entom. Zeitung, XXXIV. Jhg., Heft VIII-X, Festschr. f. Reitter (22. Okt. 1915). 
wenn auch kleiner Basalgrübchen des Halsschildes, etwas abweichend gebildete Hintertibien des $\sigma^{\nearrow}$ etc. zu unterscheiden.

Rötlichbraun, die Fühler, Beine und Mundteile heller gelbbraun, mit mäßig langer, nahezu anliegender, gelblicher Behaarung bekleidet. Kopf mäßig groß, auf der Oberseite nur mit sehr spärlicher, äußerst undeutlicher, auch unter starker Lupenvergrößerung schwer sichtbarer Punktierung. Augen groß, gewölbt. Fühler etwa die halbe Körperlänge erreichend, ziemlich schlank, ihr zweites Glied etwas kürzer als das erste, um mehr als die Hälfte länger als breit, das dritte, vierte und fünfte Glied untereinander fast gleich gestaltet, etwa um die Hälfte länger als breit, das sechste und siebente Glied eben merklich kürzer als die vorhergehenden, etwas länger als breit oder fast quadratisch, das achte Glied etwas kürzer als das siebente, etwa so breit wie lang, die dreigliedrige Fühlerkeule wenig deutlich abgesetzt, das neunte Fühlerglied etwa so breit wie lang, das zehnte leicht transversal, das Endglied der Fühler fast so lang wie die beiden vorhergehenden zusammengenommen, zugespitzt. Halsschild etwa im vorderen Viertel am breitesten, daselbst ungefähr so breit wie lang, sehr viel breiter als der Kopf, auf der Scheibe ziemlich stark gewölbt, mit spärlicher, feiner, sehr undeutlicher Punktierung, vor der Basis mit melireren sehr kleinen, aber deutlichen punktförmigen Grübchen, vor der Mitte der Basis mit Andeutung einer schwachen, gegen die Seiten zu erlöschenden Transversalfurche, innerhalb des Seitenrandes im hinteren Drittel jederseits mit seichter Längsfurche. Flügeldecken sehr langgestreckt-eiförmig, fast zweieinhalbmal so lang wie der Halsschild, mit wenig dichter, aber grober und tiefer Punktierung, mit wenig großer, seichter, nach rückwärts in eine undeutliche Furche verlängerter Skutellargrube und sehr seichter, kurzer Intrahumeralfurche. Beim o sind die Schenkel der Vorderbeine beträchtlich, jene der Mittel- und Hinterbeine schwächer keulenförmig verdickt, die Hintertibien fast gerade, nur im distalen Fünftel oder Sechstel leicht nach einwärts gebogen, in ihrer distalen Hälfte leicht verbreitert, in der Mitte des Innenrandes mit einem großen, dreieckigen, am Ende abgestumpften Zahn, an der inneren Apikalecke mit einem geraden, nach rückwärts gerichteten Endsporn.

L 0 ng. : $1 \cdot 2-1 \cdot 4 \mathrm{~mm}$.

Calabrien, von Herrn G. Paganetti-Hummler bei Sta. Eufemia d'Aspromonte in wenigen Exemplaren entdeckt. 


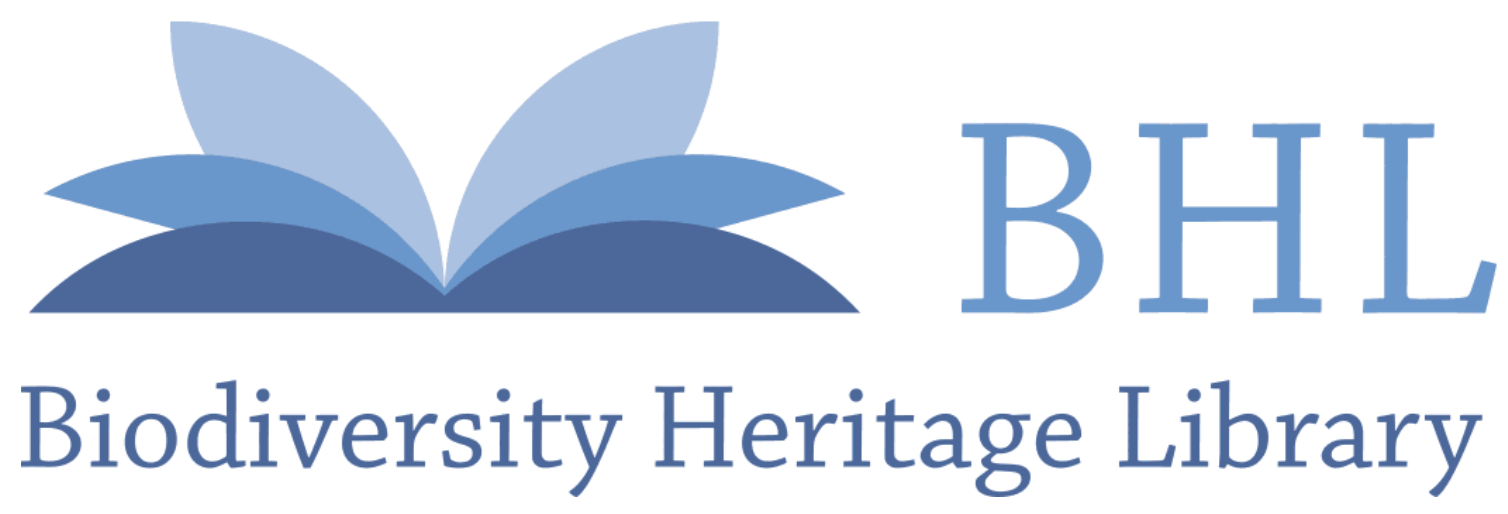

Holdhaus, Karl. 1915. "Nachträge und Berichtigungen zum Verzeichnis der Coleopteren des Monte Gargano." Wiener entomologische Zeitung 34, 349-352. https://doi.org/10.5962/bhl.part.10627.

View This Item Online: $\underline{\text { https://www.biodiversitylibrary.org/item/43833 }}$

DOI: https://doi.org/10.5962/bhl.part.10627

Permalink: https://www.biodiversitylibrary.org/partpdf/10627.

\section{Holding Institution}

Smithsonian Libraries

\section{Sponsored by}

Smithsonian

\section{Copyright \& Reuse}

Copyright Status: NOT_IN_COPYRIGHT

This document was created from content at the Biodiversity Heritage Library, the world's largest open access digital library for biodiversity literature and archives. Visit BHL at https://www.biodiversitylibrary.org. 